\title{
LA LEY DE PROTECCIÓN DEL MEDIO MARINO: HACIA LA GOBERNANZA MARÍTIMA
}

\author{
Mercè ORTIZ GARCÍA \\ Profesora titular de Derecho Administrativo \\ Departamento de Estudios Jurídicos del Estado. Universidad de Alicante \\ merce.ortiz@ua.es
}

Recibido: 20 de junio de 2011 / Aceptado: 13 de octubre de 2010

RESUMEN: Este trabajo tiene como objeto dar a conocer la Ley 41/2010, de 29 de diciembre, de Protección del Medio Marino, fruto de la transposición de la Directiva Marco sobre la Estrategia Marina, que ha sido, además, aprobada en calidad de legislación básica en el Estado español en materia ambiental. Dicha Ley viene a dotar al espacio marino español de un marco regulador integrador muy ambicioso, pues, por una parte, persigue la gestión de todos los sectores marítimos respetando el ecosistema marino donde se desarrollan — lo que se conoce como enfoque ecosistémico-, y, por otra, persigue la protección del medio marino en su totalidad, es decir, tanto el volumen acuático marino como su biodiversidad. Y para lograr tal ambicioso objetivo, la Ley se ha dotado de unas herramientas muy eficientes como son, fundamentalmente, las llamadas "estrategias marinas", escenario esencial de la planificación espacial y temporal, y la "Red de Áreas Marinas Protegidas", que, sin duda, potenciará el gran valor ambiental per se de las áreas marinas protegidas.

RESUM: Aquest treball té com objecte donar a conèixer la "Llei 41/2010, de 29 de desembre, de protecció del medi marí", fruit de la transposició de la Directiva marc sobre l'estratègia marina, que ha estat, a més a més, aprovada en qualitat de legislació 
bàsica a l'Estat espanyol en matèria ambiental. Aquesta Llei dóna a l'espai marí espanyol d'un marc regulador integrador molt ambiciós, doncs d'una banda, persegueix la gestió de tots els sectors marítims respectant l'ecosistema marí on es desenvolupen el que es coneix com enfocament ecosistèmic_-, i d'altra banda, persegueix la protecció del medi marí en la seva totalitat, és a dir, tant el volum aquàtic marí com la seva biodiversitat. I per a aconseguir tal ambiciós objectiu la Llei s'ha dotada d'unes eines molt eficients com són, fonamentalment, les anomenades "estratègies marines", escenari essencial de la planificació espacial i temporal, i la "xarxa d'àrees marines protegides", que, sens dubte, potenciarà el gran valor ambiental per se de les àrees marines protegides.

ABSTRACT: This study has as object "the protection of the marine environment Act 2010", in application of marine strategy framework Directive, which has been, in addition, approved as basic legislation in the Spanish State in environmental matter. The above mentioned Law comes to provide to the marine Spanish space of a regulatory of integration very ambitious frame, so on the one hand, chases the management of all the maritime sectors respecting the aquatic ecosystem where they develop - that is known as ecosystem approach - , and on the other hand, the protection of the marine environment chases in its entirety, that is to say, both the aquatic marine volume and his biological diversity. And to achieve such an objective self-seeker the Law it has been provided with a few very efficient tools since it are, fundamentally, the so called "marine strategies", essential scene of the spatial and temporary planning, and the "network of marine protected areas", which, undoubtedly, will promote the great environmental value per se of the marine protected areas.

PALABRAS CLAVE: enfoque ecosistémico — estrategia marina — red de áreas marinas protegidas — biodiversidad — contaminación marina.

PARAULES CLAU: enfocament ecosistèmic — estratègia marina — xarxa d'àrees marines protegides — biodiversitat — contaminació marina. 
KEYWORDS: ecosystem approach — marine strategies - network of marine protected areas — biological diversity — marine pollution.

Sumario: I. Consideraciones previas. II. El ámbito espacial de aplicación. III. El objeto de la Ley de Protección del Medio Marino. 1. La protección de todo el medio marino y su integración general. IV. El régimen competencial. V. Las estrategias marinas: instrumentos esenciales de planificación. VI. La Red de Áreas Marinas Protegidas de España. VII. Sobre los vertidos en el mar. VIII. Conclusiones. IX. Bibliografía

\section{CONSIDERACIONES PREVIAS}

Los mares y océanos representan el 99\% del espacio vital disponible de nuestro planeta, cubren el $71 \%$ de la superficie de la Tierra y contienen el $90 \%$ de la biosfera, es decir, más diversidad biológica que los ecosistemas terrestres y de agua dulce. De igual modo, la superficie marítima de las zonas bajo jurisdicción de los Estados miembros de la Unión Europea es mayor que su superficie terrestre ${ }^{1}$, albergando además un valioso patrimonio natural, respecto al cual España tiene una gran responsabilidad dada su gran biodiversidad marina.

Además, los océanos son los auténticos pulmones de la Tierra —nuestra fuente principal de oxígeno-, desempeñando un papel determinante en las condiciones climáticas.

Pero el mar, cada día más, es objeto de un uso intensivo — pesca, transporte marítimo, prospección de gas y petróleo, etc.-, en un espacio que cada vez se hace más vulnerable dadas las grandes presiones a que está sometido constantemente contaminación por sustancias peligrosas, introducción de especies exóticas, destrucción de hábitats, ruido submarino-, además de dejarse notar los efectos derivados del cambio climático.

España es un país eminentemente marítimo no solo cuantitativamente, pues la superficie marina del país duplica la terrestre ${ }^{2}$, sino también cualitativamente, dadas las

\footnotetext{
${ }^{1}$ Las aguas marinas bajo la soberanía y jurisdicción de los Estados miembros de la Unión Europea comprenden las aguas del mar Mediterráneo, del mar Báltico, del mar Negro y del noroeste del océano Atlántico, incluidas las aguas alrededor de las Azores, Madeira y las Canarias. Las aguas marinas europeas se dividen en cuatro regiones (con subregiones, en su caso): el mar Báltico, el océano Atlántico Nororiental, el mar Mediterráneo y el mar Negro. En cada región y posibles subregiones a que pertenezcan, los Estados miembros interesados deberán coordinar su acción entre sí y con los terceros Estados interesados.

${ }^{2}$ En efecto, el espacio marino suma algo más de un millón de kilómetros cuadrados de aguas marinas.
} 
consideraciones geográficas, económicas y ecológicas. En efecto, España cuenta con aproximadamente $8.000 \mathrm{~km}$ de costa, repartidos entre una península y dos archipiélagos, bañados el norte y suroeste por el océano Atlántico, y la costa este y sureste por el mar Mediterráneo. Asimismo, posee un total de diez comunidades autónomas (en adelante, CC. AA.) costeras, a saber: Galicia, Asturias, Cantabria, País Vasco, Cataluña, Comunidad Valenciana, Murcia, Islas Baleares e Islas Canarias, a las que hay que sumar las ciudades autónomas de Ceuta y Melilla. En este territorio costero viven de forma estable unos 23 millones de personas, es decir, el 58\% de la población total española vive dentro del $15 \%$ de la superficie del territorio nacional que representan nuestras costas. Estos datos demuestran, entre otras cosas, la gran relevancia de las zonas costeras en relación con su medio marino adyacente ${ }^{3}$.

En sintonía con esa gran densidad se observa que el entorno marino constituye un factor decisivo para la prosperidad económica, el bienestar social y la calidad de vida debido fundamentalmente a la gran cantidad de actividades que cobija, como la pesca - España es la principal potencia pesquera de Europa-, el turismo, la navegación, las instalaciones de producción energética, etc.

Además, España tiene —como ya anunciamos - una gran responsabilidad, pues disfruta del mayor patrimonio natural de Europa, tanto terrestre como marino.

Recapitulando, se puede afirmar que el medio marino constituye una riqueza inestimable, cosa que se ha sabido desde muy antiguo, como lo demuestran los escritos ${ }^{4}$ que nos dejaron los sumerios — considerada la primera civilización-, que explican que el mundo que conocían surgió del llamado "mar infinito", creándose todo lo que veían "del barro del mar océano", que para ellos era el que alimentaba el universo y también la fuente de vida.

\footnotetext{
${ }^{3}$ Como he hecho hincapié en repetidas ocasiones, por ejemplo: ORTIZ GARCÍA, M., La gestión eficiente de la zona costera. Los parques marinos, Tirant lo Blanch, Valencia, 2003; ORTIZ GARCÍA, M., "Las áreas marinas protegidas y la ordenación sostenible e integrada del litoral", en Estudios sobre la ordenación, planificación y gestión del litoral: Hacia un modelo integrado y sostenible (F. J. SANZ LARRUGA, dir.; M. GARCÍA PÉREZ, coord.), Fundación Pedro Barrié de la Maza-Instituto de Estudios Económicos de Galicia, A Coruña, 2009.

${ }^{4}$ Como muy elocuentemente nos cuenta D. COMPANY SEVA en Apuntes sobre los orígenes de nuestra civilización. Recuperado el 10 de noviembre de 2011 de http://hdl.handle.net/10045/19191. Es muy significativo que desde los "primeros escritos" de la humanidad aparezca ya el medio marino y con esas relevantes atribuciones; y decimos bien "los primeros escritos", pues la civilización de Sumer inventó la escritura.
} 
A pesar de esa temprana sabiduría, hemos tardado muchísimo en valorar y, por lo tanto, en ordenar y proteger el medio marino, y en su contexto oportuno, es decir, de forma integrada. Prácticamente hay que situarse en la última década del siglo XX; anteriormente se habían adoptado regulaciones sobre el medio marino, pero sectoriales, sin una visión de conjunto que integrara las múltiples actividades que se dan cita en el mar - sin colisionar entre ellas y sin deteriorar el ecosistema marino que les sirve de base-

En efecto, entre los primeros atisbos de esa sabia visión encontramos en el seno de Naciones Unidas la Agenda 21, en concreto su capítulo 17 (dedicado a los mares), donde se determina que el medio marino, a saber, los océanos, todos los mares y las zonas costeras adyacentes, constituye un todo integrado que es un componente esencial del sistema mundial de sustentación de la vida y un valioso recurso que ofrece posibilidades para un desarrollo sostenible.

En la actualidad y desde la Unión Europea existe claramente un amplio consenso en torno a la necesidad de gestionar los mares de forma holística, como lo demostró el VI Programa de Acción Comunitario en materia de Medio Ambiente ${ }^{5}$, que ofrece el marco para el establecimiento en el plano comunitario de una política marítima integrada, identificando la protección del medio marino como una de sus áreas prioritarias.

A partir de ese marco, la Unión Europea ha dado grandes muestras de buen hacer para establecer "una política marítima integrada" a través de multitud de documentos ${ }^{6}$ que, aunque por lo general no son jurídicamente vinculantes, han ido preparando el camino para la adopción de la Directiva 2008/56/CE, por la que se establece un marco de acción comunitaria para la política del medio marino ${ }^{7}$ (conocida como la Directiva Marco sobre la Estrategia Marina), que introduce la obligación de lograr un buen estado ambiental de las aguas marinas europeas mediante la elaboración de lo que denomina "estrategias marinas", con el objetivo final de preservar la biodiversidad de los océanos y mares europeos a la vez que posibilita su aprovechamiento sostenible.

\footnotetext{
5 Adoptado mediante la Decisión 1600/2002/CE del Parlamento Europeo y del Consejo, de 22 de julio de 2002 (DOCE L 242, de 10 de septiembre de 2002).

${ }^{6}$ Como "La Gestión integrada de las zonas costeras", aprobada mediante la Recomendación 2002/413/CE del Parlamento Europeo y del Consejo, de 30 de mayo de 2002. En 2006 se adopta el libro verde "Hacia una futura política marítima para la Unión: perspectiva europea de los océanos y mares”, COM(2006) 275 final, de 7 de junio de 2006. Y en 2007, el libro azul "Una política marítima integrada para la Unión Europea", COM(2007) 575, de 10 de octubre de 2007.

${ }^{7}$ Publicado en el DO L 164 de 25 de junio de 2008.
} 
No hay que olvidar que el medio marino es el recurso base para todas las actividades económicas marítimas. Esta directiva marco pretende asegurar que el desarrollo de las múltiples actividades que se dan cita en el mar no ponga en peligro la salud del ecosistema marino. De ahí que se diga que esta directiva constituye el "pilar medioambiental" de la política marítima integrada.

La Ley 41/2010, de 29 de diciembre, de Protección del Medio Marino (en adelante, Ley de Protección del Medio Marino), la norma objeto de nuestra atención, incorpora al derecho español ${ }^{8}$ la citada Directiva.

La Ley de Protección del Medio Marino viene a dotar al medio marino español de un marco regulador coherente que garantice su buen estado ambiental. España posee legislación específica y sectorial de aplicación en el mar que la presente Ley no pretende modificar ni derogar. Se trata, por ejemplo, de la legislación sobre navegación, puertos, pesca marítima, hidrocarburos, lucha contra la contaminación marina, protección de especies y hábitats, entre otras. De hecho, las estrategias marinas que se aprueben al amparo de la presente Ley reforzarán la aplicación de la legislación sectorial aplicable al medio marino para garantizar su articulación coherente y coordinada, de manera que las actividades humanas en el mar no comprometan su buen estado ambiental.

En definitiva, la Ley de Protección del Medio Marino establece un marco jurídicamente vinculante para la ordenación y gestión de todo el espacio marítimo español —algo inédito y muy necesario en nuestro ordenamiento jurídico-, como el que ya existe - $\mathrm{y}$ desde hace tiempo- para el espacio terrestre.

\section{EL ÁMBITO ESPACIAL DE APLICACIÓN}

La Ley de Protección del Medio Marino será de aplicación a todas las aguas marinas, incluidos el lecho, el subsuelo y los recursos naturales, sometidas a soberanía o jurisdicción española (art. 2.2). Más en concreto, se entienden incluidos en estas aguas ${ }^{9}$ tanto el mar territorial como la zona económica exclusiva del océano Atlántico (incluido

\footnotetext{
${ }^{8}$ Con un poco de retraso, pues el límite temporal establecido por la Directiva Marco de la Estrategia Marina era el 15 de julio de 2010.

${ }^{9}$ En España diversas normas regulan la jurisdicción en los espacios marinos; a saber, la Ley 10/1977, de 4 de enero, sobre mar territorial, y la Ley 15/1978, de 20 de febrero, sobre la zona económica exclusiva.
} 
el mar Cantábrico) ${ }^{10}$ y la plataforma continental ${ }^{11}$, así como la zona de protección pesquera para el Mediterráneo ${ }^{12}$.

Respecto a las aguas costeras ${ }^{13}$, dado que la aplicación de la Directiva Marco del Agua en España ya contempla la garantía de la consecución del buen estado de esas aguas, la Ley de Protección del Medio Marino solo se aplicará en los aspectos de la protección o la planificación del medio marino que no se hayan contemplado en los planes hidrológicos de cuenca en España, como, por ejemplo, el establecimiento de áreas marinas protegidas (AMP, en adelante), la protección de especies marinas amenazadas o el control de los vertidos desde buques o aeronaves. Por lo tanto, solo se aplicará a las aguas costeras, incluido el dominio público portuario, si la regulación derivada de la Directiva Marco del Agua no es suficiente para garantizar el buen estado ambiental de esta parte del medio marino (disposición adicional quinta).

De esta manera, la Ley de Protección del Medio Marino se centrará en la parte marina del dominio público marítimo-terrestre, definida en la Ley 22/1988, de 28 de julio, de Costas (art. 3), que era la asignatura pendiente de nuestro ordenamiento jurídico, pues la Ley de Costas se ciñe, fundamentalmente, a la parte terrestre del dominio público marítimo-terrestre.

Asimismo, se tendrán en cuenta los efectos transfronterizos sobre la calidad del medio marino de terceros países situados en la misma región o subregión marina (art. 13.4).

\section{EL OBJETO DE LA LEY DE PROTECCIÓN DEL MEDIO MARINO}

\footnotetext{
${ }^{10}$ Respecto al mar Mediterráneo, todavía no se ha declarado la zona económica exclusiva ni en España ni en el resto de Estados ribereños por falta de acuerdos entre Estados vecinos para establecer las equidistancias oportunas, ya que la falta de espacio imposibilita el establecimiento íntegro de dicha zona, es decir, las 200 millas marinas.

${ }^{11}$ Incluida la plataforma continental ampliada que España puede obtener en aplicación del procedimiento previsto en el artículo 76 del Convenio de Derecho de Mar.

${ }^{12}$ Que se extiende desde el límite del mar territorial al sur de Punta Negra-Cabo de Gata, continuando al este hasta la línea equidistante con los países ribereños, hasta la frontera marítima con Francia. Esta zona fue establecida para suplir la ausencia de la zona económica exclusiva en el Mediterráneo mediante el Real Decreto 1315/1997, de 1 de agosto, modificado por el Real Decreto 431/2000, de 31 de marzo.

${ }^{13}$ Definidas como "las aguas superficiales situadas hacia tierra desde una línea cuya totalidad de puntos se encuentra a una distancia de una milla náutica mar adentro desde el punto más próximo de la línea de base que sirve para medir la anchura de las aguas territoriales y que se extienden, en su caso, hasta el límite exterior de las aguas de transición" (artículo 16 bis del texto refundido de la Ley de Aguas, aprobado por Real Decreto Legislativo 1/2001, de 20 de julio).
} 
El propósito de la Ley de Protección del Medio Marino, como ya se ha esbozado, es el logro de un buen estado ambiental del medio marino ${ }^{14}$; medio marino que es, además, considerado en toda su extensión, es decir, referido tanto al volumen acuático como a la biodiversidad que contiene, como lo evidencian los descriptores para determinar el buen estado ambiental (recogidos en el anexo II) y asimismo la variedad de medidas proteccionistas que recoge la Ley, a saber, el control de la contaminación marina, las AMP, la protección de especies amenazadas... Esta cuestión del contenido del ambiente marino, en absoluto baladí, será desarrollada infra .

Siendo rigurosos, habría que señalar que la Ley de Protección del Medio Marino conlleva la ordenación del espacio marítimo, que no marino, pues quiere gestionar todos los sectores relacionados con el mar de manera integrada, es decir, respetando los ecosistemas.

Siguiendo con el objeto de la Ley, cabe señalar que la herramienta para alcanzar dicha meta es una planificación coherente de las actividades que se llevan a cabo en el medio marino. Actividades que hay que entender en sentido amplio, pues no solo se refieren a los sectores económicos que interaccionan en el medio marino, sino también a las acciones proteccionistas, tales como las declaraciones de AMP, la protección de especies amenazadas, la prevención y lucha contra la contaminación marina, etc.

Esa planificación será realidad mediante las conocidas estrategias marinas. Las estrategias marinas - como veremos con mayor detalle infra- son los instrumentos esenciales de la planificación del medio marino (art. 1.3).

Asimismo, aparece como objeto de la Ley, dada la naturaleza jurídica del espacio marino como bien de dominio público ${ }^{15}$, "asegurar un uso sostenible de los recursos del medio marino que tenga en consideración el interés general" (art. 1.2). Se hace referencia a dos cuestiones clave, a saber: el uso sostenible y el interés general.

Esa caracterización de "bien común" la encontramos de nuevo en otro precepto que señala que "la utilización de las aguas marinas, incluidos el lecho, el subsuelo y los recursos naturales, será libre, pública y gratuita para los usos compatibles con su

\footnotetext{
${ }^{14}$ De ahí que la Ley de Protección del Medio Marino no se aplicará a las actividades cuyo único propósito sea la defensa o la seguridad nacional. Aunque el Estado se esforzará por garantizar que dichas actividades se lleven a cabo, en la medida en que ello sea razonable y factible, de un modo compatible con el medio marino (art. 2.4).

${ }^{15}$ Como expresamente hará hincapié la Ley (arts. 1.2, 2.1, 3.1).
} 
naturaleza de bien de dominio público, de conformidad con lo establecido en la actual Ley de Costas (art. 31), y con la preservación de su integridad” (art. 3.1.).

Y de nuevo: "Fuera del uso común general ${ }^{16}$ descrito anteriormente no se admitirán sobre el medio marino más derechos de uso, explotación y aprovechamiento que los autorizados en virtud de la legislación sectorial aplicable, que se planificarán de acuerdo con la estrategia de la demarcación marina correspondiente o de manera que sean compatibles con ésta” (art. 3.2).

Se hablará de la toma en consideración de las normativas sectoriales, en particular aquellas vinculadas al interés general (art. 5.1.g).

Otro aspecto muy destacable en relación con la gestión de los bienes comunes es la gran presencia de los poderes públicos, y hay que decir que la Ley que comentamos se hace eco de ello a lo largo del texto articulado, donde además se fomenta la coordinación y cooperación entre todas las administraciones involucradas (art. 22). Asimismo, es imprescindible contar con la participación pública, que será recogida en la Ley al posibilitar a los ciudadanos presentar sus observaciones respecto a los principales trámites de las estrategias y sus actualizaciones (art. 21) ${ }^{17}$.

La gestión del medio marino como bien colectivo, común, de dominio público debe incorporar a todas las partes, y muy especialmente a los usuarios del mar, que, entendemos, deberían tener una mención específica que no tienen.

Se va a requerir, por lo tanto, la colaboración de todas las administraciones con competencias en el medio marino, incluso a nivel internacional (art. 4.1 d), así como la participación activa de organizaciones, colectivos (científicos, académicos, usuarios) y ciudadanía en general en los procesos de elaboración de las "estrategias marinas".

En definitiva, queremos hacer hincapié en que la Ley de Protección del Medio Marino incide en las pautas para gestionar correctamente los bienes comunes; que se está, por lo tanto, en el buen camino de la gestión del medio marino para evitar así "la tragedia de los comunes" que denunciaba Garret ${ }^{18}$, y más recientemente Ostrom, premio Nobel de

\footnotetext{
${ }^{16}$ La cursiva es nuestra.

${ }^{17}$ Como son: la evaluación inicial y la definición del buen estado ambiental, los objetivos ambientales, los programas de seguimiento, los programas de medidas y la actualización de las estrategias marinas (art. 21.1).

${ }^{18}$ En "The tragedy of the commons", Science, núm. 3859/1968, pp. 1243 y ss.
} 
Economía precisamente por indagar en la necesaria e importante gestión de los bienes comunes ${ }^{19}$.

En síntesis, con esta ley se puede conseguir la deseada gobernanza ${ }^{20}$ marítima $^{21}$, que se verá a su vez reforzada, entre otros motivos, por los principios que la respaldan. El primero de ellos, el principio del desarrollo sostenible, a pesar de las críticas que pueda recibir, todavía nos sirve como concepto y referente de la Ley, pues es aquel desarrollo que garantiza "que las actividades y usos en el medio marino sean compatibles con la preservación de su biodiversidad" (art. 1.3c).

Inmediatamente hemos de referirnos al principio o enfoque ecosistémico, el otro gran referente de la Ley, es decir, el enfoque global integrador de las actividades marítimas que responda a las necesidades del ecosistema. El enfoque ecosistémico implica ordenar las actividades marítimas de manera integrada y respetando los ecosistemas. Conlleva, en definitiva, que se consideren tanto los aspectos ambientales como los socioeconómicos.

Y aquí se pone de manifiesto otro acierto de la Ley, a saber, la necesidad de análisis de costes y beneficios para la protección del medio marino (art. 5.2), que no es de extrañar dado el extenso espacio marino, cuyo conocimiento todavía es incompleto; dado el catálogo de medidas contenidas en la Ley, que se traducen en unos costes desconocidos y también cuantiosos; y dados los costes que supone el deterioro de este medio (art. 8.3c).

\footnotetext{
${ }^{19}$ Vid. sobre su excelente aportación en OSTROM, E., El gobierno de los bienes comunes, Fondo de Cultura Económica, México, 2009.

${ }^{20}$ Hablamos de gobernanza porque la participación pública desempeña un papel destacado y porque, siguiendo a CANALES ALIENDE, el Gobierno deberá actuar conforme a los siguientes principios: 1) la percepción de la legitimidad; 2) la importancia central del papel de los ciudadanos; 3) la visión de un proyecto sobre la sociedad en la que actúa, y 4) la adaptación de la gestión pública; en CANALES ALIENDE, J. M., "Gobernabilidad y gestión pública", B. Olías de Lima (coord.), La nueva gestión pública, Prentice Hall, Madrid, 2001, p. 38. Asimismo, se puede consultar mi aportación a la materia en "Gobernanza y sostenibilidad", en Revista de estudios de la Administración Local (REAL) núm. 289, mayo-agosto de 2002, pp. 91 y ss., donde se justifica la necesidad de una gestión pública eficiente, haciendo hincapié precisamente en el concepto de gobernabilidad o gobernanza. Asimismo, es oportuna la consulta del "Libro blanco sobre la gobernanza europea" (Documento COM (2001) 428 final).

${ }^{21}$ Como ha sido manifiestamente requerida desde la Unión Europea en la Comunicación de la Comisión: "Orientaciones para un planteamiento integrado de la política marítima: hacia mejores prácticas de gobernanza marítima integrada y consulta de las partes interesadas". COM (2008) 395 final.
} 
A su vez — como se evidencia - esta ley tiene que ser un acicate para la investigación marina $^{22}$ (vid. arts. 4.1.g), 12, 30).

El enfoque ecosistémico ya es un clásico en la política marítima y, además, actúa como megaprincipio, pues da entrada a otros muchos, a saber: el principio de precaución, el principio de acción preventiva, el principio de corrección en la fuente, el principio de "quien contamina, paga"23 (art. 4). Y todo ello en aras de conseguir un aprovechamiento sostenible del mar (de sus bienes y servicios).

\section{La protección de todo el medio marino y su integración general}

En este momento vamos a detenernos en una cuestión conceptual que nos va a servir para conocer mejor la utilidad y el alcance de la Ley que comentamos.

En la actualidad sabemos que el bienestar del mar depende tanto de los elementos ambientales (la columna de agua, el lecho marino y el subsuelo) como de los recursos biológicos que contiene, puesto que todos ellos contribuyen al mantenimiento del equilibrio ecológico ${ }^{24}$. Ambas vertientes son importantes y se necesitan.

Nuestra tesis es que el marco normativo que estamos revisando es global, es decir, versa tanto sobre los elementos ambientales como sobre la biodiversidad, como lo demuestran, respectivamente, las medidas de control de vertidos y las AMP y su red.

Todo esto que comentamos está directamente relacionado con el contenido del concepto jurídico de ambiente, cuestión que en la actualidad es prácticamente pacífica, pero que vamos a retomar porque ayudará a entender mejor - como decimos - el objeto ambicioso de la Ley.

En un principio fueron señaladas definiciones muy extensas de ambiente, tanto que lo llegaban a identificar con el conjunto de elementos físicos, psíquicos y sociales que condicionan la vida del ser humano; de esta manera, el ambiente lo era todo y, por lo tanto,

\footnotetext{
${ }^{22}$ En igual sentido se manifiesta E. ALONSO GARCÍA en "La reciente e inminente legislación sobre conservación y uso sostenible de la biodiversidad del medio marino ¿celebración o reto?", en Ambienta, febrero de 2011, p. 73.

${ }^{23}$ Para conocer bien estos principios, vid. LOPERENA ROTA, D., Los principios del derecho ambiental, IVAP-Organismo Autónomo del Gobierno Vasco, Civitas, Madrid, 1998, p. 79.; asimismo, GARCÍA URETA, A., Derecho europeo de la biodiversidad, Iustel, Madrid, 2010, pp. 123 y ss.

${ }^{24} \mathrm{Y}$ todos estos elementos o vertientes conforman la noción amplia del derecho ambiental. Sobre este particular y aplicado al ambiente marino, vid. mi monografía: La conservación de la biodiversidad marina: Las áreas marinas protegidas, Comares, Granada, 2002, pp. 199 y ss.
} 
un concepto tan amplio no tenía ninguna utilidad para el Derecho. En sentido contrario se encontraban autores como Martín Mateo, que mantenían un concepto restringido de ambiente. Para este autor, el ambiente quedaría reducido a "los elementos naturales de titularidad común y características dinámicas: en definitiva, el agua y el aire, vehículos de transmisión, soporte y factores esenciales para la existencia del hombre sobre la tierra" ${ }^{25}$. Este concepto restringido excluía claramente la conservación de la naturaleza ${ }^{26}$. Otra corriente doctrinal mantenía que, junto a las técnicas de lucha contra la contaminación, se debía tener en cuenta de alguna manera la protección de la naturaleza ${ }^{27}$.

En la actualidad parece que la postura de identificar el ambiente también con la naturaleza se ha impuesto, sobre todo desde la Conferencia de Río de Janeiro de $1992^{28}$, que hizo hincapié en la conservación de la diversidad biológica mundial ${ }^{29}$.

Por lo tanto, en la actualidad prevalece una definición jurídica de ambiente relativamente extensa, puesto que la acción pública ambiental no se conforma con el control de la contaminación —que coincidiría con la definición restrictiva de ambiente—, sino que comprende también la protección de los recursos vivos ${ }^{30}$, aunque sin llegar a una concepción omnicomprensiva del ambiente, como se predicaba en un principio por parte de cierta doctrina.

Sin embargo, López Ramón considera útil conceder alguna virtualidad a esa primera concepción amplísima del ambiente, que, recordemos, llegaba a identificar el ambiente prácticamente con “todo". En efecto, esa concepción puede “configurar la protección del

\footnotetext{
${ }^{25}$ Dentro de esta concepción restringida del ambiente habría autores que añadirían un tercer gran sistema: el suelo.

${ }^{26}$ Vid. MARTÍN MATEO, R., Tratado del Derecho Ambiental, vol. I, Trivium, Madrid, 1991, pp. 80 y ss.

${ }^{27}$ LAMARQUE, J. et al., Droit de la protection de la nature et de l'environment, LDGI, París, 1973, p. XV.

${ }^{28}$ El propio Martín Mateo, influido en parte por la "sensibilidad biológica" de Río 1992, ha optado por esta comprensión amplia del ambiente, aunque mantiene que "las técnicas utilizadas para proteger la naturaleza viva y los sistemas naturales son radicalmente diferentes", en Tratado del Derecho Ambiental, vol. III, Trivium, Madrid, 1997, pp. 21-23.

${ }^{29}$ De acuerdo con las investigaciones más recientes, que ponen de relieve el importante papel de los elementos biológicos para el mantenimiento del equilibrio ecológico, y no solo, por lo tanto, el de los elementos abióticos.

${ }^{30}$ Como afirma Machado Carrillo, que muy gráficamente se refiere a estos dos componentes del ambiente como la "línea azul", es decir, la lucha contra la contaminación, y la "línea verde", ligada a la conservación de la naturaleza y al uso de los recursos naturales, con la finalidad de garantizar el desarrollo sostenible y el mantenimiento de la biodiversidad. De esta manera, el mismo autor dirá que, frente al tradicional principio "azul" de "quien contamina, paga", se instaura, si no explícitamente, sí de facto, el nuevo principio "verde" de "quien conserva, gana". En MACHADO CARRILLO, A., Los biólogos y la gestión de áreas marinas protegidas, Colegio Oficial de Biólogos, publicación núm. 10, Madrid, 1992, p. 6.
} 
medio ambiente, desde el punto de vista jurídico, como una finalidad, como un gran objetivo del ordenamiento aplicable a todas las políticas públicas ${ }^{31 " ;}$ es decir, "no debería tratarse de englobar las diferentes políticas públicas en una sola política ambiental, sino de imprimir un impulso protector del medio ambiente en todos los sectores y líneas de actuación pública"32.

La protección del ambiente tendría, pues, "dos grandes contenidos; uno, general, que afecta a todas las políticas públicas, exigiendo que éstas tengan en cuenta la situación de los recursos afectados; y otro específico, que se ocupa de velar directa y sectorialmente por la utilización racional de los recursos naturales". Y ese contenido específico a su vez se divide — como dijimos - en la protección de la parte abiótica y en la conservación de la naturaleza viva.

Pues bien, ese "contenido general" coincidiría con el tan mencionado enfoque ecosistémico $^{33}$ de la Ley que comentamos, o lo que es lo mismo, la dimensión general y horizontal de la protección ambiental, como lo evidencia la Directiva Marco sobre la Estrategia Marina cuando, entre otras cosas, quiere "promover la integración de las consideraciones medioambientales en todas las políticas pertinentes y proporcionar el pilar medioambiental para la futura política marítima de la Unión Europea" (preámbulo, párrafo 3), y que se sustancia en las "estrategias marinas". No en vano dichas estrategias constituyen el marco general al que deberán ajustarse necesariamente las diferentes políticas sectoriales y actuaciones administrativas con incidencia en el medio marino de acuerdo con lo establecido en la legislación sectorial correspondiente y con las características del ecosistema marino.

Mientras que el "contenido específico" del ambiente coincidiría con el enfoque sectorial y vertical que la Ley de Protección del Medio Marino recoge de forma íntegra, como ya ha quedado dicho, su protección se sustancia, entre otras medidas, en el control de los vertidos y las AMP, que constituyen los ejemplos paradigmáticos de lo que conforma en la actualidad el contenido específico del concepto jurídico de ambiente.

\footnotetext{
${ }^{31}$ La cursiva es nuestra.

${ }^{32}$ LÓPEZ RAMÓN, F., "El derecho ambiental como derecho de la función pública de protección de los recursos naturales"..., op. cit., p. 108.

${ }^{33} \mathrm{Y}$ en el llamado principio de integración, al respecto vid. GARCÍA URETA, A., Derecho europeo de la biodiversidad..., op. cit., pp. 132-134.
} 
Para acabar de centrar correctamente la Ley, cabe señalar que la función pública de protección ambiental utiliza dos caminos diferentes, como bien aprecia Loperena Rota. Por una parte, la Administración actúa directamente sobre el medio (atmósfera, aguas, suelo o biodiversidad) y, por otra, actúa sobre las causas de degradación (productos tóxicos, agricultura, residuos, biotecnología o radiactividad $)^{34}$. Pues bien, la Ley de Protección del Medio Marino comprende las dos vertientes, dadas las variadas actuaciones que conlleva.

\section{EL RÉGIMEN COMPETENCIAL}

La Ley de Protección del Medio Marino se dicta "al amparo del artículo 149.1.23. a de la Constitución Española, que establece la competencia del Estado sobre legislación básica sobre protección del medio ambiente, sin perjuicio de las facultades de las Comunidades Autónomas de establecer normas adicionales de protección del medio ambiente en su territorio" (disposición final primera) ${ }^{35}$.

Como es sabido, para el derecho europeo el responsable ante la Unión Europea es siempre el Estado unitariamente entendido, con independencia de quien tenga internamente la competencia.

Los Estados miembros legislan sobre sus áreas marítimas y aplican y ejecutan la mayor parte de las políticas sectoriales en el mar. La Unión Europea solo tiene competencia exclusiva sobre pesca, mientras que respecto al resto de las políticas que inciden en el mar comparte las competencias con los Estados miembros. Por lo tanto, en la materia que nos atañe el protagonismo recae en los Estados miembros de acuerdo con el principio de subsidiariedad, mientras que la Unión Europea facilitará la integración ${ }^{36}$.

Pero dada la idiosincrasia del medio marino — su gran conectividad-, los Estados situados en una misma región marina tendrán la obligación de coordinar sus acciones y aplicarán sus políticas marítimas con una visión global. Se recomienda para ello el recurso a los mecanismos de cooperación que establecen los convenios internacionales vigentes; las organizaciones internacionales derivadas de dichos convenios proporcionarán sus competencias científicas y técnicas, y permitirán ampliar la

\footnotetext{
${ }^{34}$ LOPERENA ROTA, D., Los principios del derecho ambiental, IVAP..., op. cit., p. 79.

${ }^{35}$ En parecidos términos, artículo 3.1.

36 Sobre la distribución competencial entre la Unión Europea y los Estados miembros en materia ambiental, vid. GARCÍA URETA, A., Derecho europeo de la biodiversidad..., op. cit., pp. 148 y ss.
} 
cooperación a los terceros países que participan en ellos. Así, se fomenta la cooperación internacional entre los Estados miembros de la Unión Europea, así como con terceros países vecinos.

Y las instancias decisorias subnacionales, como son en España las comunidades autónomas (CC. AA., en adelante) tienen un gran peso ${ }^{37}$, pues, además de beneficiarse del planteamiento integrado, son las que corren más riesgos cuando faltan actuaciones globales.

No hay que olvidar cuál es el objetivo de esta normativa, a saber: una planificación integral de actividades sin comprometer el medio marino que las alberga. Y el instrumento de planificación por excelencia son las estrategias marinas, en cuya elaboración deben participar tanto el Estado como las CC. AA., por cuanto estas ejercen competencias sobre las políticas sectoriales marítimas, que deberán adaptarse a los objetivos de las estrategias. Por lo tanto, el establecimiento de políticas integradas no debe generar una centralización; en definitiva, una política integrada no debe ser la excusa para modificar el sistema competencial vigente.

La totalidad de las aguas españolas recaen bajo la jurisdicción de la Administración general del Estado ${ }^{38}$. Las otras administraciones territoriales, CC. AA. y municipios, carecen de espacio marino, aunque sí ejercen determinadas competencias en el medio marino ${ }^{39}$, localizadas fundamentalmente en las aguas interiores, jurisdicción a la que no afecta la Ley de Protección del Medio Marino. También ejercen competencias fuera,

\footnotetext{
${ }^{37}$ Las competencias sobre los asuntos marítimos y costeros se comparten entre la Administración general del Estado y las CC. AA. de acuerdo con la distribución de competencias prevista en la Constitución de 1978 (arts. 148 y 149), así como en los respectivos estatutos de autonomía.

${ }^{38}$ Canarias, desde la aprobación de su Estatuto de Autonomía (1982), ha tratado de que se incorporaran al territorio insular las aguas interinsulares, en términos jurisdiccionales, algo equivalente a las aguas archipelágicas. Esta opción está basada en una confusa redacción de la Ley de Zona Económica Exclusiva española, que establece que el límite exterior de la ZEE se medirá desde las líneas de base recta que unen los puntos extremos de las islas como si se aplicara el régimen archipelágico. En octubre de 2010 el Gobierno de la nación llegó a un acuerdo con Canarias para que las aguas interinsulares fueran definidas como "aguas canarias", aunque sin precisar cuál iba a ser su estatuto jurídico con arreglo al Convenio sobre el Derecho del Mar.

${ }^{39}$ Sobre el principio de territorialidad de las competencias, puede consultarse ORTIZ GARCÍA, M., La conservación de la biodiversidad marina: Las áreas marinas protegidas..., op. cit., pp. 610 y ss. Asimismo, VALENCIA MARTÍN, G., “¿De quién es el mar?: La distribución de competencias entre el Estado y las Comunidades Autónomas en materia de protección del medio marino”, F. Sosa Wagner (coord.), El Derecho Administrativo en el umbral del siglo XXI. Libro homenaje a Ramón Martín Mateo, Tirant lo Blanch, Valencia, 2000, pp. 3610 y ss. También es muy recomendable la consulta de GIMÉNEZ CASALDUERO, M., "Las áreas marinas protegidas: nuevas perspectivas a la luz de la Ley 42/2007, del patrimonio natural y de la biodiversidad", Revista Catalana de Dret Ambiental, vol. I - núm. 1, 2010, pp. 131. Recuperado el 30 de octubre de 2011 de http://rcda.cat/index.php/rcda/article/view/6.
} 
más allá de las aguas interiores, como sucede en las AMP de su competencia — como indicaremos infra-, en materia de cultivos marinos... Pero las competencias autonómicas pueden alcanzar mayor protagonismo, ya que muchas de las actividades que hay que controlar para conseguir el buen estado medioambiental del medio marino están localizadas en tierra.

La Ley garantiza la participación de las CC. AA. en la elaboración de las estrategias marinas y en el seguimiento de su aplicación, en la medida en que estas afecten a sus competencias, a través de la creación de los comités de seguimiento de las estrategias marinas para cada una de las "demarcaciones marinas" — unos determinados ámbitos espaciales como veremos infra-, que estarán integrados por representantes de las administraciones estatal y autonómica con competencias en la ejecución de la estrategia correspondiente. Asimismo, perfila los mecanismos de coordinación y cooperación necesarios para alcanzar los objetivos de protección del medio marino en un marco de pleno respeto a las competencias atribuidas a las CC. AA.

Se atribuye al Gobierno de la nación la competencia para establecer unas directrices que garanticen la coherencia en el conjunto de la planificación. El Gobierno podrá aprobar directrices comunes a todas las estrategias marinas con el fin de garantizar la coherencia de sus objetivos en aspectos tales como: la Red de Áreas Marinas Protegidas de España; los vertidos en el mar; los aprovechamientos energéticos situados en el medio marino; la investigación marina y el acceso a los datos marinos; la evaluación y el seguimiento de la calidad ambiental del medio marino; la ordenación de las actividades que se llevan a cabo en el medio marino o pueden afectarle, y la mitigación de los efectos del cambio climático y la adaptación a este (art. 4.2).

En cualquier caso, hay que reconocer que la Ley de Protección del Medio Marino solo crea un limitado aparato institucional cuyo nivel administrativo es ministerial: la Comisión Interministerial de Estrategias Marinas y los comités de seguimiento de las estrategias marinas (art. 22). El resto de las instituciones son órganos de coordinación ya existentes entre la Administración general del Estado y las CC. AA. (Conferencia Sectorial de Medio Ambiente). En resumen, podría decirse que se trata de una labor del Estado, aunque con participación autonómica.

Asimismo, como ha quedado dicho, se garantiza un proceso de participación pública por el que los ciudadanos pueden presentar sus alegaciones en relación con las 
estrategias marinas, y que se basa en la obligación ${ }^{40}$ de poner a disposición del público resúmenes sobre los elementos del "plan de acción" para la elaboración de las estrategias marinas al objeto de que los ciudadanos puedan presentar sus observaciones (art. 21). Esta participación debe ser real, sin impedimentos ni excusas por parte de las administraciones si se quiere conseguir una gobernanza eficaz.

\section{LAS ESTRATEGIAS MARINAS: INSTRUMENTOS ESENCIALES DE PLANIFICACIÓN}

Las estrategias marinas constituyen el marco general al que deberán ajustarse necesariamente las diferentes políticas sectoriales y actuaciones administrativas con incidencia en el medio marino, de acuerdo con lo establecido en la legislación sectorial correspondiente (art. 7), y respetando al ambiente marino, de acuerdo con el enfoque ecosistémico que irradia toda la Ley de Protección del Medio Marino.

Las estrategias pretenden evaluar cómo los proyectos realizados en la costa y en el mar afectan al medio marino, así como organizar de manera racional los diferentes usos (transporte marítimo, pesca, acuicultura, actividades receptivas...).

En definitiva, las estrategias tienen como objetivo proteger y restablecer los ecosistemas marinos europeos, y garantizar la viabilidad ecológica de las actividades económicas relacionadas con el medio marino de aquí al año 2021.

Y para poder conseguir tan loable objetivo, las estrategias marinas conllevarán la realización de una serie de tareas consecutivas — que además se deben circunscribir a unos determinados ámbitos espaciales, llamados “demarcaciones marinas”establecidas por la Ley de Protección del Medio Marino (art. 6) ${ }^{41}$.

Las demarcaciones marinas responden a unas subdivisiones de las distintas regiones y subregiones en que se divide el medio marino español de acuerdo con la Ley de Protección del Medio Marino. En efecto, el medio marino español se divide en región del Atlántico nororiental (subregión del golfo de Vizcaya y las costas ibéricas, y subregión Atlántico macaronésica de Canarias) y región del mar Mediterráneo.

\footnotetext{
${ }^{40}$ De conformidad con la Ley 27/2006, de 18 de julio, por la que se regulan los derechos de acceso a la información, de participación pública y de acceso a la justicia en materia de medio ambiente.

41 Asimismo, se habilita al Gobierno para definir nuevas demarcaciones marinas o modificar las existentes (disposición adicional segunda).
} 
A efectos de la presente Ley y para facilitar la aplicación de las estrategias marinas sobre las anteriores regiones y subregiones marinas — como ya se ha avanzado-, se establecen las demarcaciones marinas (art. 6.2).

En total, la Ley establece cinco demarcaciones marinas; tres en la región del Atlántico (noratlántica, sudatlántica y canaria) y dos en la del Mediterráneo (levantino-balear y Estrecho y Alborán), que han sido definidas teniendo en cuenta las particularidades biogeográficas, oceanográficas e hidrológicas de cada región.

Avanzando con las estrategias marinas, recalcamos que estas conllevan un conjunto de pasos consecutivos, con calendario para su realización ${ }^{42}$, que culminan en un programa de medidas que vamos a pasar a revisar.

La primera tarea es la evaluación inicial del estado del medio marino ${ }^{43}$ (plazo julio de 2012) - a iniciativa del Ministerio de Medio Ambiente y Medio Rural y Marino, previa consulta de los departamentos ministeriales y las CC. AA. costeras-, que incluye las características naturales, las presiones y los impactos y un análisis económico y social de la utilización del medio marino. El anexo I incluye una lista indicativa de las características, las presiones y los impactos a tener en cuenta en esta evaluación.

A continuación, la segunda tarea es la determinación de lo que se llama el buen estado ambiental $^{44}$ de las aguas (plazo julio de 2012), a saber: "aquel en el que éste da lugar a océanos y mares ecológicamente diversos y dinámicos, limpios, sanos y productivos en el contexto de sus condiciones intrínsecas, y en el que la utilización del medio marino sea sostenible, quedando así protegido su potencial de usos, actividades y recursos por parte de las generaciones actuales y futuras" (art. 9.1).

El Ministerio de Medio Ambiente y Medio Rural y Marino, previa consulta a las CC. AA. y en función de la evaluación inicial, definirá para cada demarcación marina un conjunto de características correspondientes al buen estado ambiental, basándose para ello en los once descriptores cualitativos enumerados en el anexo II, como son, por

\footnotetext{
${ }^{42}$ Recogido en la disposición adicional cuarta.

${ }^{43}$ Se entiende por estado medioambiental el estado general de las aguas marinas, teniendo en cuenta la estructura, el funcionamiento y los procesos de los ecosistemas que componen el medio marino, los factores fisiográficos, geográficos, biológicos, geológicos y climáticos naturales, y las condiciones físicas, acústicas y químicas que se derivan sobre todo de la actividad humana interna o externa en la zona en cuestión (art. 8.2).

${ }^{44}$ Para facilitar a los Estados miembros la coherencia en las tareas de definición del "buen estado ambiental", se ha aprobado la Decisión de la Comisión, de 1 de septiembre de 2010, sobre los criterios y las normas metodológicas aplicables al buen estado ambiental de las aguas marinas.
} 
ejemplo, la diversidad biológica, la presencia de especies alóctonas, la salud de las reservas, la red trófica, la eutrofización, la modificación de las condiciones hidrográficas y la concentración de contaminantes, la cantidad de residuos o la contaminación acústica (art. 9.2).

La tercera tarea es la propuesta por parte del Ministerio de Medio Ambiente y Medio Rural y Marino del establecimiento de una serie de objetivos ambientales (e indicadores), teniendo en cuenta la lista indicativa de características del anexo III, enfocados a lograr el buen estado ambiental (plazo julio de 2012) que previamente se ha definido respecto de cada demarcación marina. Al mismo tiempo se deben definir una serie de indicadores para poder evaluar la consecución de los objetivos ambientales (art. 10.1 y 2$)$.

Esta propuesta, junto con la definición del buen estado ambiental, será sometida a acuerdo del Consejo de Ministros previa consulta a los organismos públicos de investigación competentes (art. 12) ${ }^{45}$ y al Consejo Asesor de Medio Ambiente, y previo informe de las CC. AA. afectadas, una vez cumplido el trámite de participación pública.

Sobre la base de la evaluación inicial, la cuarta tarea consiste en que el Ministerio de Medio Ambiente y Medio Rural y Marino establezca un programa de seguimiento (plazo julio de 2014), de acuerdo con las orientaciones del anexo IV, para evaluar periódicamente el estado de las aguas y el cumplimiento de los objetivos que se hayan definido (art. 11).

En último lugar, una vez aprobados los objetivos ambientales y teniendo en cuenta la evaluación inicial, el Ministerio de Medio Ambiente y Medio Rural y Marino, en colaboración con las CC. AA. y el resto de administraciones públicas implicadas, elaborará un programa de medidas (plazo 2015 para la redacción y 2016 para la puesta en marcha) para lograr el buen estado ambiental. El anexo $\mathrm{V}$ incluye una lista no exhaustiva de los tipos de medidas que podrán adoptar estos programas, que tendrán una duración de seis años; entre otras, destacan las medidas de control y reducción de la contaminación (título IV), las AMP (título III), los planes de recuperación y conservación de especies marinas (también en el título III), etc. (arts. 13 y 14).

\footnotetext{
${ }^{45}$ Como el Instituto Español de Oceanografía, destacado por la propia Ley de Protección del Medio Marino; no en vano es una institución con una prestigiosa trayectoria que se configura en la actualidad como un organismo público de investigación, de acuerdo con la Ley 13/1986, de 14 de abril, de Fomento y Coordinación General de la Investigación Científica y Técnica.
} 
Cuando la puesta en marcha del programa de medidas implique la actuación de otras administraciones públicas, estas informarán al Ministerio de Medio Ambiente y Medio Rural y Marino de las disposiciones o actuaciones que se lleven a cabo en cumplimiento de las estrategias marinas (art. 16).

Las estrategias marinas, que incluirán el programa de medidas, deberán ser aprobadas por el Consejo de Ministros mediante real decreto, previo debate en el seno de la Conferencia Sectorial de Medio Ambiente y previo informe de los ministerios y las comunidades autónomas afectados y del Consejo Asesor de Medio Ambiente, una vez cumplido el trámite de información pública (art. 15).

Cuando el Ministerio de Medio Ambiente y Medio Rural y Marino determine que el estado del medio marino de una zona específica es crítico, el Gobierno adoptará medidas urgentes, avanzando la ejecución de los programas de medidas, incluyendo medidas de protección más estrictas (art. 17.1).

Para cada una de las estrategias marinas, el Ministerio de Medio Ambiente y Medio Rural y Marino, mediante consultas e informes previos, deberá especificar las razones que impidan la realización de algunas de esas medidas (acción u omisión de otro Estado, fuerza mayor, etc.), es decir, las excepciones al cumplimiento de la estrategia, siguiendo las pautas establecidas en la Ley (art. 18).

Todos los elementos de las estrategias marinas se deben actualizar al menos cada seis años (art. 20). Hay que tener presente el carácter dinámico de los sistemas marinos, el hecho de que las presiones y los impactos que se ejercen sobre ellos pueden variar según se modifiquen las actividades humanas, y otras agresiones, como las derivadas del cambio climático. También hay que tener en cuenta los avances de los conocimientos sobre el medio marino, que son el resultado de los progresos científicos y tecnológicos. Todo ello justifica la constante necesidad de actualizar.

Corresponde a la Unión Europea, en concreto a la Comisión, garantizar la coherencia de las acciones de los Estados miembros, los cuales deberán presentarle los datos sobre los elementos de sus estrategias en cada etapa de su elaboración. Esos datos son evaluados por la Comisión, que puede orientar a los Estados para garantizar así el cumplimiento de la estrategia y la coherencia de las medidas contempladas (art. 12, Directiva Marco sobre la Estrategia Marina). 


\section{LA RED DE ÁREAS MARINAS PROTEGIDAS DE ESPAÑA}

El título III de la Ley de Protección del Medio Marino, que es el que vamos a revisar, se centra en las AMP, más en concreto en la Red de AMP, aunque también se refiere a la conservación de especies $^{46}$, en desarrollo de la Ley 42/2007, de 13 de diciembre, del Patrimonio Natural y de la Biodiversidad (en adelante, Ley de la Biodiversidad), para adaptarlo a las estrategias marinas (art. 28, apdos. h), i).

Y es que dentro de la protección de la naturaleza — que es donde nos situamos en estos momentos - cabría realizar otra clasificación, pues la legislación aplicable en esta parcela se perfila desde una doble perspectiva: sectorial y territorial; en la perspectiva sectorial se incardina la conservación de especies, y en la territorial se encontrarían las AMP. La normativa de AMP, referida a enclaves territoriales, contempla estas zonas de manera integral y atendiendo a la totalidad de los recursos presentes y usos posibles; en cambio, la normativa sectorial - como la aplicable a la flora y fauna (a las especies marinas, en nuestro caso) - atiende únicamente al recurso natural que constituye en cada caso su objeto, con independencia de que se sitúe en un espacio protegido o fuera de él. Esta última fue la primera estrategia conservacionista en aparecer y actualmente ha perdido protagonismo dadas las bondades que se predican de la protección de las especies en sus respectivos hábitats, en nuestro caso, en las AMP.

La Ley de Protección del Medio Marino completa en esta materia la Ley de la Biodiversidad, que es la norma que da base a la protección de la biodiversidad marina en España.

España, a través de la Ley de la Biodiversidad, crea la figura de área marina protegida como una de las categorías de espacios naturales protegidos ${ }^{47}$ y determina que estas áreas se integrarán en la Red de Áreas Marinas Protegidas. Hasta ahora, la Red no se había regulado; la presente Ley crea formalmente la Red de Áreas Marinas Protegidas de España y establece cuáles son sus objetivos, los espacios naturales que la conforman y los mecanismos para su designación y gestión.

\footnotetext{
${ }^{46}$ Precisamente se acaba de aprobar el Real Decreto 139/2011, de 4 de febrero, para el desarrollo del Listado de Especies Silvestres en Régimen de Protección Especial y del Catálogo Español de Especies Amenazadas, y se objeta el escaso número de especies marinas incluidas.

${ }^{47}$ Sobre la caracterización de las AMP en la Ley de la Biodiversidad, se puede consultar mi publicación "La protección del medio marino", en Noticias de la Unión Europea, núm. 307, 2010, pp. 55 y ss.
} 
Antes de tratar estas cuestiones, vamos a explicar brevemente estos espacios naturales y para ello traemos a colación la definición que hace de ellos la Ley de la Biodiversidad, a saber: "Las Áreas Marinas Protegidas son espacios naturales designados para la protección de ecosistemas, comunidades o elementos biológicos o geológicos del medio marino, incluidas las áreas intermareal y submareal, que en razón de su rareza, fragilidad, importancia o singularidad, merecen una protección especial. Podrán adoptar esta categoría específica o protegerse mediante cualquier otra figura de protección de áreas prevista en esta Ley, en cuyo caso, su régimen jurídico será el aplicable a estas otras figuras, sin perjuicio de su inclusión en la Red de Áreas Marinas Protegidas" (art. 32.1).

Además, con la Ley de Protección del Medio Marino — dadas las "interconexiones" que caracterizan al medio marino- la protección de la biodiversidad marina mediante AMP puede ser muy efectiva, pues dicha Ley tiene muy presente las causas de la reducción de la biodiversidad en origen, a saber: la contaminación marina y la sobreexplotación pesquera $^{48}$.

Dado el carácter ambiental de la Ley de Protección del Medio Marino, la Red de Áreas Marinas Protegidas de España constituye una pieza fundamental para la conservación de la biodiversidad a largo plazo mediante la designación, protección y conservación de las áreas clave, las áreas prioritarias para las especies, los hábitats y los procesos ecológicos que albergan, estableciendo los mecanismos para una gestión responsable.

Si son muchas las bondades que se predican de las $\mathrm{AMP}^{49}$, más aún lo serán de la Red, como muestra la propia Ley de Protección del Medio Marino al hablar de sus objetivos, a saber, "asegurar la conservación y recuperación del patrimonio natural y la biodiversidad marina; proteger y conservar las áreas que mejor representan el rango de distribución de las especies, hábitats y procesos ecológicos en los mares; fomentar la conservación de corredores ecológicos y la gestión de aquellos elementos que resulten esenciales o revistan primordial importancia para la migración, la distribución geográfica y el intercambio genético entre poblaciones de especies de fauna y flora marinas" (art. 25).

\footnotetext{
${ }^{48}$ El enfoque comunitario garantiza la coherencia entre sectores y con las demás políticas europeas, como la política pesquera común o la política marítima europea.

${ }^{49}$ Sobre el concepto y la caracterización de las AMP in extenso, se puede consultar mi monografía $L a$ conservación de la biodiversidad marina: Las áreas marinas protegidas..., op. cit., pp. 91 y ss.
} 
Además, hay que tener presente que España debe cumplir los numerosos compromisos internacionales adquiridos respecto a las redes europeas y paneuropeas que, en su caso, se establezcan, así como con la Red Global de Áreas Marinas Protegidas para el año 2012, creada por la Cumbre Mundial sobre Desarrollo Sostenible, que se celebró en Johannesburgo en 2002 en el marco del Convenio sobre la Diversidad Biológica. Esta última estrategia de conservación de la biodiversidad mundial presta una atención muy considerable a la protección de las áreas marinas ${ }^{50}$.

La Directiva Marco sobre la Estrategia Marina viene a respaldar el compromiso de la Unión Europea en el contexto del Convenio sobre la Biodiversidad ${ }^{51}$ de crear, de aquí al 2012, una red mundial de zonas marinas protegidas (párrafo 18, preámbulo). Se deben incluir medidas de protección especial que contribuyan a la constitución de redes coherentes y representativas de zonas marinas protegidas, incluyendo ZEPA, ZEC y zonas marinas protegidas en virtud de acuerdos internacionales o regionales.

Y sobre la base de la información facilitada por los Estados miembros en 2014, la Comisión presentará un informe de los progresos en la creación de zonas marinas protegidas (art. 21). En este sentido, cabe señalar que España debe aumentar en gran medida su ritmo de protección para poder cumplir sus obligaciones ${ }^{52}$.

Fruto de todo este contexto es el título III de la Ley de Protección del Medio Marino, que lleva por título "Red de áreas marinas protegidas de España y conservación de especies y hábitats marinos". La Red estará constituida por espacios protegidos situados

\footnotetext{
${ }^{50}$ En efecto, existe una gran preocupación por la conservación y el uso sostenible de la biodiversidad marina y costera, por lo que la Conferencia de las Partes (CoP) del Convenio sobre la Biodiversidad acordó en 1995 —en su segunda reunión - un programa de acción denominado "El mandato de Yakarta de diversidad biológica marina y costera" (Decisión II/10).

${ }^{51}$ Anteriormente la Unión Europea, en el marco del VI Programa de Acción Comunitario en materia de Medio Ambiente, al establecer las áreas prioritarias para la protección del medio ambiente hizo un llamamiento para desarrollar siete estrategias temáticas, incluyendo una para el medio marino dentro del área prioritaria sobre la biodiversidad. El objetivo de esta estrategia es "promover la utilización sostenible de los mares y proteger los ecosistemas marinos", prestando especial atención a los lugares que mantienen un alto valor de biodiversidad. El VI Programa de Acción también contemplaba el apoyo a la Directiva Aves y a la Directiva Hábitats, haciendo un llamamiento para la promoción de la protección de áreas marinas a través de la red Natura 2000.

52 Sobre nuestra aportación protegida y asimismo las AMP ya declaradas, vid. mi publicación "La protección del medio marino", en Noticias de la Unión Europea..., op. cit., págs. 56 y ss. En cualquier caso, en el momento presente las AMP establecidas en el "medio marino español" constituyen — como decimos - un número escaso, tanto que el área marina El Cachucho, que el 11 de noviembre de 2011, mediante Real Decreto, fue declarada como $\mathrm{AMP}^{52}$, es tildada de primera AMP, aunque realmente lo que sucede es que por el momento es la única exclusivamente marina. Se encuentra localizada en el mar Cantábrico, a 65 kilómetros de Ribadesella, Asturias. El Cachucho es una montaña submarina de más de 4 kilómetros de altura y con una superficie semejante a los Picos de Europa.
} 
en aguas marinas españolas, representativos del patrimonio natural marino español, con independencia de que su declaración y gestión estén reguladas por normas internacionales, comunitarias, estatales y autonómicas (art. 24).

En efecto, esta red se va a nutrir de áreas marinas protegidas de diversas procedencias, tanto desde un punto de vista competencial, a saber, estatal y autonómico, como material, a saber, biodiversidad y pesca. De esta manera, la Administración estatal —a través del Ministerio de Medio Ambiente y Medio Rural y Marino, y más en concreto, de la Secretaría General del Mar — declarará AMP — mediante real decreto (art. 27.1) — cuando se trate de espacios, hábitats o áreas críticas situados en áreas marinas bajo soberanía o jurisdicción nacional, con excepción de los casos en que exista continuidad ecológica $^{53}$ del ecosistema marino con el espacio natural terrestre objeto de protección (art. 6, Ley de la Biodiversidad), que serán de competencia autonómica (art. 36.1, Ley de la Biodiversidad), como lo ratifica la Ley que comentamos (art. 26.3).

Por razón de la materia, en concreto la "protección de la biodiversidad" conformará el grueso de la Red, no en vano las AMP son una estrategia ambiental. En este sentido, la Red estará conformada por las AMP del Estado (art. 26.1.a), las zonas especiales de conservación (ZEC), procedentes de la Directiva Hábitats, y las zonas de especial protección para las aves (ZEPA), procedentes de la Directiva Aves (art. 26.1.b).

También pueden formar parte de la Red otras áreas marinas protegidas existentes en el ordenamiento español a partir de la mera ratificación y publicación de tratados internacionales o la asunción de compromisos internacionales; estas áreas pueden ser reservas de la biosfera, lugares Patrimonio Mundial de la Unesco, humedales Ramsar, zonas especialmente protegidas de importancia para el Mediterráneo establecidas en el Convenio de Barcelona, y áreas marinas protegidas del Convenio OSPAR, entre otras (art. 26.1.d).

Al amparo del título de intervención "Pesca", también se integrarán en la Red las "reservas marinas" reguladas en la legislación pesquera; así, de acuerdo con su atribución competencial (arts. 148.1. 11 y 149.1.19 CE), las localizadas en aguas

\footnotetext{
${ }^{53}$ Desafortunada expresión e "invención" del Tribunal Constitucional en su STC 38/2002, de 14 de febrero. Para conocer más sobre la citada expresión, vid. mi aportación "La protección del medio marino", en Noticias de la Unión Europea..., op. cit., pp. 64-71.
} 
interiores serán autonómicas (art. 26.3), mientras que las localizadas en aguas exteriores serán estatales (art. 26.1.e) ${ }^{54}$.

Teniendo en cuenta la posible inclusión en la Red de Áreas Marinas Protegidas de espacios cuya declaración y gestión corresponden a las administraciones autonómicas, la Ley prevé para estos casos que las CC. AA. litorales, en colaboración con la Administración general del Estado, elaboren la propuesta de criterios mínimos comunes, unas directrices para una gestión coordinada y coherente de la Red que aprobará la Conferencia Sectorial de Medio Ambiente (art. 27.2).

A la Administración general del Estado le compete coordinar, seguir y evaluar la Red; redactar sus directrices comunes; proponer instrumentos de cooperación para la consecución de los objetivos de la Red; proponer a las instituciones europeas y órganos internacionales la inclusión de redes internacionales de los espacios marinos de la Red susceptibles de incardinarse en sus respectivas redes; aprobar y aplicar las estrategias y los planes de recuperación de especies marinas incluidas en el Catálogo Español de Especies Amenazadas; elaborar una memoria anual de seguimiento de las actuaciones de la Red y los informes trienales de la Red, y representar a España en las redes internacionales de AMP (art. 28).

Con carácter de instrumento básico de coordinación para la consecución de los objetivos de la Red se aprobará el Plan Director de la Red de Áreas Marinas Protegidas de España — mediante real decreto-, con un procedimiento con participación pública y con las CC. AA. litorales. Tendrá una vigencia de diez años y recogerá los objetivos en materia de cooperación con otras instancias, tanto en el ámbito nacional como internacional, las directrices para la planificación y conservación de las AMP, los proyectos de interés general... (art. 29).

El Instituto Español de Oceanografía será considerado el organismo científico de referencia para el seguimiento de las AMP y, en consecuencia, de la Red que conforman, sin perjuicio de los organismos que las CC. AA. elijan para apoyarse en la gestión de sus respectivos espacios protegidos (arts. 27.4 y 30).

\footnotetext{
${ }^{54}$ De acuerdo con la Ley 3/2001, de 26 de marzo, de Pesca Marítima del Estado (art. 14), que en breve será reemplazada por la denominada Ley de Pesca Sostenible, que en la actualidad se encuentra en tramitación en el Congreso.
} 


\section{SOBRE LOS VERTIDOS EN EL MAR}

El otro aspecto paradigmático y pionero de la protección ambiental, como es sabido, lo ocupa la prevención y lucha contra la contaminación marina, y la Ley que comentamos se hace eco también de esta dimensión ambiental a través del control de los vertidos en el mar (título IV), ya que, además, constituye uno de los objetivos específicos de las estrategias marinas (art. 1.3b).

La Ley de Protección del Medio Marino tiene un concepto muy amplio de contaminación del medio marino, de ahí que contemple todo tipo de vertidos, comenzando por los procedentes de tierra firme (art. 31); en este sentido, una política marítima integrada va más allá de la simple coordinación de las políticas marítimas, ya que un porcentaje muy relevante de la contaminación marina procede de las actividades terrestres.

Se realiza una remisión a la normativa específica, que estaría encabezada por la actual Ley de Costas, "sin perjuicio de las facultades de las CCAA de establecer normas adicionales de protección del medio ambiente en su territorio", variando la autoridad marítima competente para autorizar el vertido según la ubicación geográfica (vid. ejemplo artículo 32.6) ${ }^{55}$. En cualquier caso, dichas autorizaciones requerirán informe previo favorable del Ministerio de Medio Ambiente y Medio Rural y Marino para determinar su compatibilidad con la estrategia marina correspondiente.

Asimismo, en función de la ubicación geográfica serían aplicables los convenios marinos regionales, a saber, el Convenio de Londres sobre la Prevención de la Contaminación del Mar por Vertimiento de Desechos y Otras Materias, el Convenio de Barcelona para la Protección del Medio Marino y la Región Costera del Mediterráneo, y el Convenio OSPAR sobre la Protección del Medio Ambiente Marino del Atlántico Nordeste.

Se establecen normas también respecto a los vertidos desde buques y aeronaves, plataformas u otras construcciones en el mar, es decir, los vertidos de mar a mar (vid. in extenso art. 32).

Entre los vertidos no prohibidos se encuentra el almacenamiento de dióxido de carbono en estructuras geológicas submarinas, de acuerdo con las condiciones establecidas (art.

\footnotetext{
${ }^{55}$ De acuerdo con la jerarquía de opciones de gestión de desechos que realiza la Ley: reducción, reutilización, reciclaje ex situ... (art. 32.9).
} 
32. apdos. 3, 4 y 5), que, sin duda, serán desarrolladas en su legislación específica, la Ley 40/2010, de 29 de diciembre, de almacenamiento geológico de dióxido de carbono.

Por otro lado, sería mejorable evitar cláusulas abiertas que permitan la autorización condicionada de vertidos radiactivos al mar (art. 32.4).

Queda prohibida, con carácter general, la incineración en el mar (art. 33), y asimismo el depósito de objetos sobre el fondo marino. Los objetos que se destinen a arrecifes artificiales serán autorizados de acuerdo con su normativa, y siempre previo informe favorable del Ministerio de Medio Ambiente y Medio Rural y Marino - como es habitual - para comprobar su compatibilidad con la estrategia marina correspondiente (art. 35).

Y respecto a la responsabilidad por daños al medio marino, se realiza otra remisión, esta vez a la Ley 26/2007, de 23 de octubre, de Responsabilidad Medioambiental (disposición adicional tercera). En este punto se echa en falta un régimen especial de responsabilidad civil dados los vertidos masivos de hidrocarburos ${ }^{56}$.

\section{CONCLUSIONES}

El balance general de la Ley es positivo, aunque algunas cuestiones son ciertamente mejorables, como ya hemos tenido ocasión de señalar a lo largo del trabajo. Como crítica de conjunto, señalaría la necesidad del aumento de la presencia de las CC. AA. y de la participación ciudadana — a través de los múltiples y diversos usuarios del mar-, lo que haría posible la consecución real de la gobernanza marítima.

Otra de las ineficiencias de la Ley de Protección del Medio Marino es su abusiva considero- subordinación o remisión a otras leyes, como ocurre con el régimen de los vertidos, las infracciones y las sanciones (art. 36), y asimismo con el relevante asunto de los recursos genéticos marinos (disposición adicional primera) ${ }^{57}$. Es decir, se entiende que algunas remisiones están justificadas de acuerdo con la idiosincrasia de las "estrategias marinas", pues, recordemos, constituyen el marco general al que deberán ajustarse necesariamente las diferentes políticas sectoriales y actuaciones

\footnotetext{
56 Tampoco hay que perder de vista los vertidos en alta mar causados por el lavado de los tanques de transporte para eludir las instalaciones portuarias y así obtener mayores beneficios económicos.

${ }^{57}$ Que, dado su gran interés, debería ser objeto de especial atención en la línea del Protocolo de Nagoya sobre Acceso a los Recursos Genéticos y Participación Justa y Equitativa en los Beneficios que se deriven de su Utilización.
} 
administrativas con incidencia en el medio marino de acuerdo con lo establecido en la legislación sectorial correspondiente, pero no prácticamente todo el contexto de la Ley.

En cualquier caso, es posible que se puedan producir conflictos de aplicación de normativas con distintos niveles de protección, en cuyo caso debería prevalecer la norma que resulte más exigente respecto de la protección ambiental de dichas aguas, algo que la Ley contempla de forma parca (vid. disposición adicional quinta). Y, asimismo, la eficacia de la Ley dependerá en gran medida de su interpretación, como sucede, por ejemplo, con la expresión de "continuidad ecológica".

Pero incluso esos defectos se mitigan si tenemos en cuenta el verdadero alcance de la norma, a saber, se trata de la primera norma española que introduce la planificación espacial marina, dando coherencia al marco institucional y normativo referido al medio marino. Con esta ley se quiere evitar el actual marco fragmentado de toma de decisiones sobre asuntos marítimos, y posibilitar así la gobernanza marítima en su contexto oportuno, esto es, el enfoque ecosistémico.

Es la primera vez que se legisla teniendo en cuenta el ecosistema marino y la interacción entre los distintos usos marítimos. El mar es considerado como un todo integrado. Con esta ley se hace justicia con el medio marino; se pone en valor el medio marino per se.

El reto de la humanidad es que la naturaleza vuelva a ser la medida de todas las cosas, su límite ${ }^{58}$. Los acelerados avances científicos de los últimos tiempos, capaces de hacer creer que la ciencia facilitará todo lo que el ser humano se proponga, incluso sustituyendo recursos naturales agotados por sucedáneos, se han terminado; los destrozos irreparables de la Naturaleza han comenzado ya... ${ }^{59}$ En otras palabras, el bienestar humano solo se puede mantener si la parte biofísica, la parte viva del planeta, está en buen estado de salud.

\footnotetext{
${ }^{58}$ La conciencia del límite estaba arraigada muy profundamente en la sabiduría clásica.

${ }^{59}$ Como lo evidencian los científicos de todo el mundo liderados por J. Rockström, del Centro de Resiliencia de la Universidad de Estocolmo; identificaron nueve procesos que consideran claves en los sistemas terrestres, los que determinaron límites planetarios que intentan establecer un "espacio operativo seguro para la humanidad". Estos límites planetarios son valores máximos que la humanidad no debe superar si se quiere evitar cambios ambientales globales catastróficos. Los nuevos procesos son: la pérdida de ozono atmosférico, la acidificación de los océanos, el uso global de agua dulce, los cambios en el uso de la tierra, la carga atmosférica de aerosoles, la contaminación química, las alteraciones del ciclo del fósforo y del nitrógeno, la tasa de pérdida de biodiversidad y el cambio climático. Estos tres últimos límites planetarios ya han sido superados. El primer avance de esta investigación fue publicado en una edición especial de la revista Nature y recientemente ha sido publicado en la revista Ecology and Society.
} 
De ahí el gran peso que las AMP tienen en la Ley de Protección del Medio Marino. No obstante, España tiene todavía pocas AMP declaradas, que representan una porción ínfima de toda nuestra superficie marina; se necesita, pues, acelerar el ritmo de protección $^{60}$, y esta norma puede ser decisiva en esta ardua y obligada tarea.

Por lo tanto, considero que hay razones más que suficientes para ser optimista respecto a la Ley que comentamos, pero, además, su adecuada aplicación constituye un reto necesario para España por los motivos que comentamos a continuación.

En primer lugar, dado el enfoque ecosistémico de la Ley, es fundamental contar con el mejor respaldo científico posible que permita conocer el estado de las aguas y de la biodiversidad marina y el impacto de las agresiones, y, en consecuencia, se pueda diseñar el mejor programa de medidas (es decir, adaptable y flexible, para reflejar las peculiaridades y las ostensibles diferencias geográficas y naturales entre las distintas costas y mares). Por lo tanto, esta Ley — como ya se ha advertido - es un acicate muy necesario para fomentar la investigación marina.

En segundo lugar, puesto que se persigue una gestión integrada, es necesario que estén involucrados todos los interesados, que son muchos y variados. De ahí otro acicate de la Ley, el participativo, que permita la cooperación y coordinación de todos en el medio marino, a saber, usuarios, ciudadanos, administraciones y países vecinos.

La aplicación del principio de subsidiariedad es la otra cara de la moneda de la participación, como elemento fundamental del planteamiento integrado, de forma que las decisiones se adopten lo más cerca posible de las personas y los sectores afectados.

No hay que olvidar, además, el avance del conocimiento sobre la base de la sabiduría tradicional de las comunidades locales, como sucede con la gestión de espacios y especies marinas, en relación, por ejemplo, con la pesca artesanal.

En tercer lugar, hay que tener muy presente el acicate economicista de la Ley, pues habla de "mares productivos" y de "análisis económico y social de la utilización del medio marino".

España se encuentra entre los primeros países europeos con mayor valor añadido generado por sus actividades económicas marítimas y por número de empleos asociados a ellas. El mar reporta más empleo que otros sectores de la economía nacional. Ello se

\footnotetext{
${ }^{60}$ Sobre estos datos, vid. ORTIZ GARCÍA, M., "La protección del medio marino", en Noticias de la Unión Europea..., op. cit., pp. 56-57.
} 
debe al peso que tienen la pesca y el turismo. Esta ley podría ser utilizada para impulsar otros sectores marítimos; por ejemplo, la industria energética marina renovable es una exquisita oportunidad para España ${ }^{61}$.

En efecto, hay que estar receptivo a los nuevos desarrollos tecnológicos, pero sin menospreciar las distintas variables tradicionales (la pesca artesanal o la reconversión de los faros, donde el turismo, por ejemplo, se puede reinventar ${ }^{62}$, etc.), y para ello es necesario apelar al conocimiento y a la innovación, en suma, a la creatividad.

En definitiva, es obvio que la buena calidad del medio marino impulsará los más variados sectores marítimos, tanto los tradicionales como los más novedosos.

La Ley de Protección del Medio Marino se perfila, pues, como la principal herramienta legislativa para la gestión de los mares españoles, pero para que sea eficaz necesita la aplicación de otras normas, así como una gobernanza sabia, es decir, un "conocimiento creativo y responsable". Por lo tanto, todo un reto, pero necesario dados los malos augurios...

\section{BIBLIOGRAFÍA}

ALONSO GARCÍA, E., "La reciente e inminente legislación sobre conservación y uso sostenible de la biodiversidad del medio marino ¿celebración o reto?”, en Ambienta, febrero de 2011.

CANALES ALIENDE, J. M., “Gobernabilidad y gestión pública”, B. Olías de Lima (coord.), La nueva gestión pública, Prentice Hall, Madrid, 2001.

COMPANY SEVA, D., Apuntes sobre los orígenes de nuestra civilización. Recuperado el 10 de noviembre de 2011 de http://hdl.handle.net/10045/19191.

GARCÍA URETA, A., Derecho europeo de la biodiversidad, Iustel, Madrid, 2010.

GARRET, H., “The tragedy of the commons”, Science, núm. 3859/1968.

\footnotetext{
${ }^{61}$ Como lo demuestra la iniciativa público-privada de la Universidad de Alicante y la empresa Biofuel Systems que ha hecho posible el primer petróleo ecológico del mundo. Se fabrica con procesos industriales rentables a partir de la captura de dióxido de carbono; un revolucionario sistema acelera el proceso de creación y fosilización de la biomasa marina (algas), que dura millones de años, y lo reduce a unos pocos días. Para más información, se puede consultar su página web: www.biopetroleo.com

${ }^{62}$ Un turismo más contemplativo que no fomente el consumo excesivo, como sucede con el llamado "safari fotográfico". En las islas Medes, en Girona, hay una reserva marina protegida que tiene menos de un kilómetro cuadrado totalmente protegido, y las visitas debidamente organizadas proporcionan hasta seis millones de euros al año, un ingreso veinte veces mayor que el que brinda la pesca.
} 
GIMÉNEZ CASALDUERO, M., "Las áreas marinas protegidas: nuevas perspectivas a la luz de la Ley 42/2007, del patrimonio natural y de la biodiversidad", Revista Catalana de Dret Ambiental, vol. I-núm. 1, 2010. Recuperado el 30 de octubre de 2011 de http://rcda.cat/index.php/rcda/article/view/6.

LAMARQUE, J. et ál., Droit de la protection de la nature et de l'environment, LDGI, París, 1973.

LOPERENA ROTA, D., Los principios del derecho ambiental, IVAP-Organismo Autónomo del Gobierno Vasco, Civitas, Madrid, 1998.

MACHADO CARRILLO, A., Los biólogos y la gestión de áreas marinas protegidas, Colegio Oficial de Biólogos, publicación núm. 10, Madrid, 1992.

MARTÍN MATEO, R., Tratado del Derecho Ambiental, vol. I, Trivium, Madrid, 1991.

— Tratado del Derecho Ambiental, vol. III, Trivium, Madrid, 1997.

ORTIZ GARCÍA, M., La conservación de la biodiversidad marina: Las áreas marinas protegidas, COMARES, Granada, 2002.

— "Gobernanza y sostenibilidad", en Revista de estudios de la Administración Local (REAL) núm. 289, mayo-agosto de 2002.

- La gestión eficiente de la zona costera. Los parques marinos, Tirant lo Blanch, Valencia, 2003.

— "Las áreas marinas protegidas y la ordenación sostenible e integrada del litoral", en F. J. Sanz Larruga (dir.), M. García Pérez (coord.), Estudios sobre la ordenación, planificación y gestión del litoral: Hacia un modelo integrado y sostenible, Fundación Pedro Barrié de la Maza-Instituto de Estudios Económicos de Galicia, A Coruña, 2009.

— "La protección del medio marino", en Noticias de la Unión Europea, núm. 307, 2010 .

OSTROM, E., El gobierno de los bienes comunes, Fondo de Cultura Económica, México, 2009.

VALENCIA MARTÍN, G., “¿De quién es el mar?: La distribución de competencias entre el Estado y las Comunidades Autónomas en materia de protección del medio marino", en F. Sosa Wagner (coord.), El Derecho Administrativo en el umbral del siglo XXI. Libro homenaje a Ramón Martín Mateo, Tirant lo Blanch, Valencia, 2000. 